\title{
Equipment
}

\section{Airway management: contents of a difficult intubation cart}

Glenn P. McGuire MD, David $\mathrm{T}$. Wong $\mathrm{MD}$

$\mathrm{D}$ ESPITE our extensive clinical experience, anesthesiologists still encounter unexpected difficult intubations. We need to be prepared with an organized plan such as the ASA algorithm ${ }^{1-3}$ for management of the difficult airway. In a "can't intubate/can't ventilate" situation, emergency methods of oxygenation and ventilation must be rapidly available.

There are many anecdotal reports of intubating 'tricks'. The ASA task force ${ }^{2}$ and a recent review ${ }^{4}$ recommend the general types of equipment that should be stocked on a difficult intubation kit. There are commercially available trays specifically designed for certain aspects of airway management.

In a university teaching hospital department, we found it necessary to organize a comprehensive difficult intubation cart due to the preferences of different anesthesiologists. Since we do not practice pediatric anesthesia, our intubation cart is designed for adult patients.

There is one clearly labeled difficult intubation cart in the operating room area and two portable kit bags. Adult and pediatric fiberoptic bronchoscopes and the Bullard laryngoscope are stored on a separate portable cart.

Our purpose is not to mention all possible airway management equipment. We hope this listing will help others formulate and organize their own difficult intubation cart. It is important that anesthesiologists and trainees be familiar with the indications and limitations of these devices. Having previously thought through the steps of emergency airway management and with the equipment readily available, the anesthesiologist will be better prepared to deal efficiently with difficult airway situations.

Equipment for airway management and intubation:

A) Nasal: - nasopharyngeal airways: SIZES \#6, \#7, \#8
(Portex) (Keene, $\mathrm{NH})$

- Endotrol blind nasal trigger endotracheal tubes: sizes \#6.0, \#7.0, \#8.0

(Mallinckrodt) Glens falls, NY)

B) Oral:

- Guedel oropharyngeal airways: sizes \#8, \#9, \#10, \#11: (Portex) (Keene, NH)

- stylets/intubating guides:

a) endotracheal tube stylet (satin tip) size 14 Fr: (Mallinckrodt) (St Louis, MO)

b) Eschmann gum elastic bougie: sizes $10 \mathrm{Fr}, 15 \mathrm{Fr}$ : (Sims) (Keene, $\mathrm{NH}$ )

c) trachlight: (Laerdal) (Stavanger, Norway)

- endotracheal tubes: cuffed sizes \#5.0, \#5.5, \#6.0, \#6.5, \#7.0, \#7.5, \#8.0:

(Mallinckrodt) (St Louis, MO)

- laryngoscope blades: (Penlon) (Abingdon, Oxon, UK)

a) curved: McCoy, left handed \#3 Macintosh, Huffman prism for Macintosh blade

b) straight- \#3 and \#4 Macintosh, \#3 Miller, \#2 Seward, \#3 and \#4 Wisconsin

- laryngoscope handles: regular length, short handle, polio (adjustable).

- laryngeal masks: (Nicosia, Cyprus)

a) sizes \#3, \#4, \#5

b) Fastrach intubating laryngeal masks: sizes \#3, \#4

- endotracheal tubes for laryngeal masks:

1) sizes \#6.0, \#6.5, \#7.0 cuffed (Mallinckrodt) (St Louis, MO)

2) size \#6.0 Sheridan LTS (large yel-

From the Department of Anesthesia, The Toronto Hospital, Western Division, EC 2-046, 399 Bathurst St, Toronto, Ontario, M5T 2 S8. Address correspondence to: Glenn P. McGuire MD. Phone: 416-603-5118; Fax: 416-603-6494.

Accepted for publication October 15, 1998. 


$$
\text { low cuff) (Argyle, NY) }
$$

- Combitube (Sheridan) (Argyle, NY)

- lung isolation:

a) bronchial blockers: Fogarty occlusion catheter: size $8 / 14 \mathrm{Fr}$ (Baxter) (Santa Ana, CA)

b) double lumen endotracheal tubes: sizes \#35, \#37, \#39, \#41 Fr

(Mallinckrodt) (St Louis, MO)

C) Cricothyroid membrane:

- transcricothyroid membrane jet ventilation:

a) intravenous catheters: sizes \#12, \#14 gauge (length: 2 inches)

b) jet ventilation hose with controller handle and Luer lock connector

- retrograde transcricothyroid membrane wire (length: $145 \mathrm{~cm}$, width: 0.035 inch) (Cook Critical Care) (Bloomington, IN)

- Melker percutaneous dilational cricothyroidotomy set (uncuffed size \#6.0 tracheostomy tube) included in set (Cook Critical Care) (Bloomington, IN)

- surgical cricothyroidotomy:

a) \#3 scalpel handle, \#11 blade, trachea retraction hook

b) size \#6.0 endotracheal tube

c) Shiley cuffed tracheostomy tubes: sizes \#4, \#6, \#8, \#10: (Mallinckrodt) (Irvine, CA)

D) Accessory equipment:$$
\text { pment: }
$$

a) confirming position of endotracheal tube Easy Cap $\mathrm{ETCO}_{2}$ detector

(Nellcor Puritan Bennett/Pleasanton, CA)

b) endotracheal tube exchange catheters:

a) with jet ventilation capability

1) Cardiomed (Gormley, Ont)

2) Cook catheter: small, medium, large (Cook Critical Care) (Bloomington, IN)

b) without jet ventilation capability

- Sheridan catheter- small,

c) oxygen delivery: medium, large (Argyle, NY)

- Laerdal manual resuscitation bag

with masks and reservoir

(Stavanger, Norway)

- oxygen tubing with nipple for connecting to oxygen wall outlet or tank

d) suction:

- endotracheal suction catheters: sizes \#10, \#12, \#14 (Benlan)

(Oakville, Ont)

- Yankauer oral suction (Bard)

(Mississauga, Ont)

- suction tubing

e) other:

- spare batteries and bulbs for laryngoscope

- bite blocks

- Abelson jaw spreader (Benson)

(Markham, Ont)

- Magill forceps (Per:lon)(Abingdon, Oxon, UK)

f) local anesthetic:

- atomizer for spraying lidocaine $4 \%$ solution

- lidocaine spray ( $10 \mathrm{mg} /$ spray)

(Astra) (Mississauga, Ont)

note: all endotracheal tubes mentioned are cuffed

\section{References}

1 Benumof JL. Management of the difficult airway. Anesthesiology 1991; 75: 1087-110.

2 Practice Guidelines for the Management of the Difficult Airway. A Report by the ASA Task Force on Management of the Difficult Airway. Anesthesiology 1993; 78: 597-602.

3 The LMA and ASA-DA algorithm. Anesthesiology 1996; 84: 686-99.

4 Crosby ET, Cooper RM, Douglas MJ, et al. The unanticipated difficult airway with recommendations for management. Can J Anaesth 1998; 45: 757-76. 